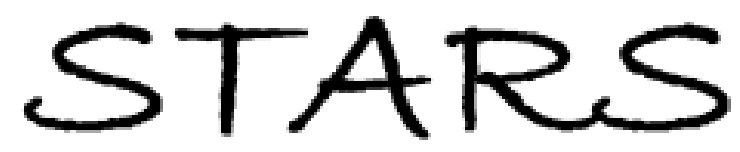

University of Central Florida

STARS

$1-1-2007$

\title{
Characterization study of bonded and unbonded polydimethylsiloxane aimed for bio-micro-electromechanical systems-related applications
}

\author{
Miao Liu \\ University of Central Florida \\ Quanfang Chen \\ University of Central Florida
}

Find similar works at: https://stars.library.ucf.edu/facultybib2000

University of Central Florida Libraries http://library.ucf.edu

This Article is brought to you for free and open access by the Faculty Bibliography at STARS. It has been accepted for inclusion in Faculty Bibliography 2000 s by an authorized administrator of STARS. For more information, please contactSTARS@ucf.edu.

\section{Recommended Citation}

Liu, Miao and Chen, Quanfang, "Characterization study of bonded and unbonded polydimethylsiloxane aimed for bio-micro-electromechanical systems-related applications" (2007). Faculty Bibliography 2000s. 7362.

https://stars.library.ucf.edu/facultybib2000/7362

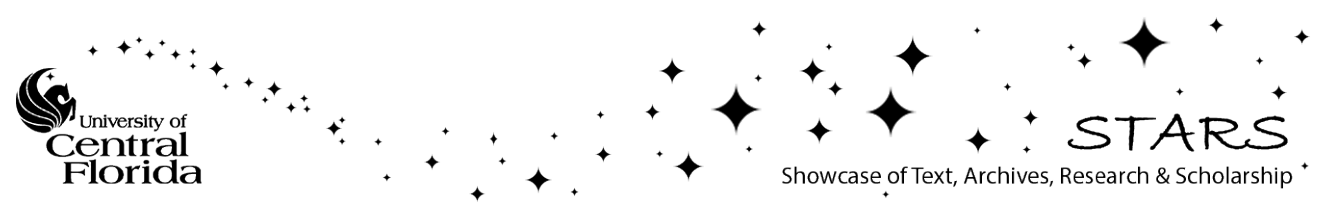




\section{Characterization study of bonded and unbonded polydimethylsiloxane aimed for bio-micro- electromechanical systems-related applications}

\author{
Miao Liu \\ Quanfang Chen \\ University of Central Florida \\ MEMS and Nanomaterials Lab, Mechanical, \\ Materials and Aerospace Engineering \\ Department \\ Orlando, Florida 32816-2450 \\ E-mail: qchen@mail.ucf.edu
}

\begin{abstract}
Authors have demonstrated that by controlling the mixing ratio of polydimethylsiloxane's (PDMS's) two components-base polymer (part $A$ ) and a curing agent (part $B$ )-different mechanical properties of PDMS can be achieved. Test results show that the Young's modulus decreases as the increasing of mixing ratios $(A: B)$. However, there is a transitional mixing ratio (part $A$ :part $B=10$ ) after which the Young's modulus is almost independent of the mixing ratio. The PDMS's thickness plays an important role in determining the mechanical properties. The results show that the thinner the PDMS, the stiffer it behaves. The bonding strength between two cured PDMS parts with different mixing ratios shows that it depends on the mixing ratio. A maximum bonding strength of $130 \mathrm{kPa}$ occurs on a bonded couple with mixing ratios of $30 A: 1 B$ and $3 A: 1 B$, respectively. The fracture on bonded specimens does not occur at the bonding interfaces. Instead it occurs at the side with a larger portion of part $A$. The intermediate material property formed at the interface is attributed to the diffusion layer formed. (๑) 2007 Society of Photo-Optical Instrumentation Engineers. [DOI: 10.1117/1.2731381]
\end{abstract}

\begin{abstract}
Subject terms: PDMS; mechanical properties; mixing ratios; bonding strength.
Paper 05067SSRR received Sep. 15, 2005; revised manuscript received Dec. 5, 2006; accepted for publication Feb. 22, 2007; published online May 11, 2007. This paper was originally scheduled for publication in the April-June 2006 special section on bio-mems and micro fluidics, but was not available in time.
\end{abstract}

\section{Introduction}

Polydimethylsiloxane (PDMS) elastomer, due to its unique properties, has been widely used in fabricating lab-on-achip (LOC) or micro total analysis systems ( $\mu$ TAS) for biological applications. PDMS is biocompatible, which is critical for biological assays. PDMS is optically transparent to wavelengths down to near $256 \mathrm{~nm}$. ${ }^{1}$ The transparent nature of PDMS is suitable for biological analysis using widely adopted methods such as fluorescence. PDMS's elastic and soft nature make it ideal for the reversible deformations needed during analytical operations. $\mu \mathrm{TAS}$ or LOC systems made of PDMS can be realized by molding casting or soft lithography with high fidelity. ${ }^{2-5}$ PDMS can seal to itself and other flat surfaces with a good watertight capability. Meanwhile, the fabrication of LOC or $\mu$ TAS or other microdevices using PDMS are cost-effective, which makes PDMS suitable for disposable uses in biomedical applications. ${ }^{6}$ As a result, PDMS is getting more and more interest as a bio-micro-electromechanical systems (bioMEMS) material, and the application of utilizing PDMS are increased greatly in a broad range of applications. For example, Young Shik Shin et al. ${ }^{7}$ succeed in fabricating a PDMS-based micro polymer chain reaction (PCR) chip using a replica modeling method. Ceriotti ${ }^{8}$ reported a $\mu \mathrm{TAS}$ system, a capillary electrochromatography microchip fabricated with PDMS consisting of a $10-\mu \mathrm{m}$ particle-free slanted "double-T" injector. PDMS has also been used to

$1537-1646 / 2007 / \$ 25.00$ ๑ 2007 SPIE fabricate a microarray for DNA analysis. Xiaomei et al. ${ }^{9}$ introduced a disposable three-dimensional (3D) microchip array for DNA amplification made of PDMS in which 1064 column chambers were fabricated.

PDMS normally contains a base polymer (part $A$ ) and a curing agent (part $B$ ). PDMS is cured by an organometallic cross-linking reaction promoted by heating, when the two parts are mixed with a certain mixing (weight) ratio. ${ }^{10}$ Very importantly, cured PDMS can be bonded together with a plasma treatment or by controlling the mixing ratios between the bonding couple. ${ }^{11}$ The capability to bond makes PDMS prevail in fabricating stacked 3D MEMS devices.

Many of PDMS-based microbiological devices require

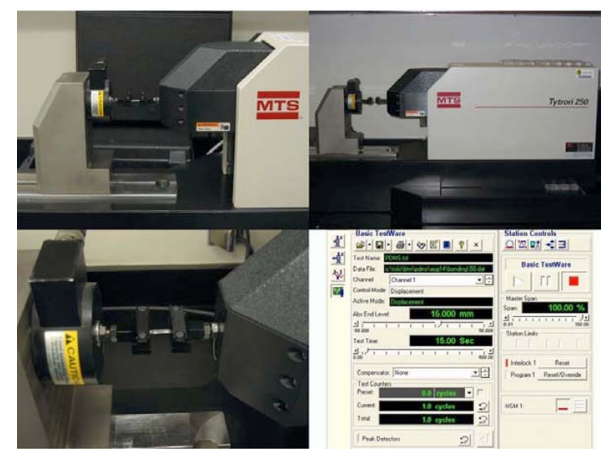

Fig. 1 MTS Tytron 250 microforce tester system (with PDMS samples mounted on two leveled clamps) and control software interface. 
Table 1 Cured RTV 615 properties.

\begin{tabular}{lcc}
\hline \hline Properties & Unit & Value \\
\hline Hardness, shore A durometer & & 44 \\
Elongation & $\%$ & 120 \\
Shrinkage & & 0.2 \\
Refractive index & $\mathrm{KV} / \mathrm{mm}(\mathrm{V} / \mathrm{mil})$ & $19.7(500)$ \\
Dielectric strength & & 2.406 \\
Dielectric constant at $1000 \mathrm{~Hz}$ & & 0.0006 \\
Dissipation factor at $1000 \mathrm{~Hz}$ & $\mathrm{Ohm}-\mathrm{cm}$ & $1.8 \times 10^{15}$ \\
Volume resistivity & deg C/cm & 0.00045 \\
Thermal conductivity & $\mathrm{cm} / \mathrm{cm}$, deg C & $27 \times 10^{-5}$ \\
Coefficient of expansion & $\mathrm{cal} / \mathrm{gm}, \mathrm{deg} \mathrm{C}$ & 0.3 \\
\hline Specific heat & & \\
\hline \hline
\end{tabular}

manipulating a PDMS membrane to realize the microfluidic management, which is the key function for biological reactions. The actuation mechanisms on PDMS include, but are not limited to, (1) pneumatic, ${ }^{11}$ (2) thermomechanical, ${ }^{12}$ and (3) hydraulic. ${ }^{13}$ To accommodate these actuations, PDMS's mechanical properties are critical to fulfill microdevices' needs. For example, the actuation force required to manipulate a PDMS membrane is proportional to PDMS's thickness and stiffness. In addition, 3D structures are realized by bonding, and the bonding strength between the bonded couple is a key factor in determining the workability as well as the reliability of the devices fabricated. Therefore, the mechanical properties of PDMS are very important, but not enough adequate data are available.

In this paper, both bonded and unbonded PDMS's mechanical properties are investigated considering the influence of varied composition, membrane thickness, and bonding conditions. PDMS preparation, the test methods, and results are presented and discussed.

\section{Experimental Preparation}

\subsection{PDMS}

Silicone RTV 615 (General Electric) was studied in this paper. Part $A$ of RTV 615 is a siloxane oligomer containing polydimethylsiloxane bearing vinyl groups and a platinum catalyst. Part $B$ of RTV 615, the cross-linking oligomers, contains a cross-linker containing silicon hydride $(\mathrm{Si}-\mathrm{H})$ groups, which form covalent bonds with vinyl groups ${ }^{10}$ when mixed with part $A$. The PDMS curing is enhanced by an additional heating process after the mixing of parts $A$ and $B$. A typical mixing ratio is around $10 A: 1 B$ in weight. Table 1 provides manufacturer's cured PDMS properties at this mixing ratio. However, mechanical properties with different mixing ratios, especially for those with large mixing ratios, which are of interest to the research community are not available.
Table 2 Specimen series of bonded couples.

\begin{tabular}{lcccc}
\hline \hline Series Number & 1 & 2 & 3 & 4 \\
\hline Ratio 1 $(A: B)$ & $20: 1$ & $30: 1$ & $40: 1$ & $50: 1$ \\
Ratio 2 $(A: B)$ & $2: 1$ & $3: 1$ & $4: 1$ & $5: 1$ \\
\hline \hline
\end{tabular}

\subsection{Bonding Methods}

Bonding has been the main approach in fabricating 3D biological assays. One way of bonding PDMS has been realized with oxygen plasma treatment, during which oxygen plasma leads to oxidation on surface, and chain scission as well as cross-linking are ensured by the formation of a silicalike surfaces. ${ }^{14}$ Another bonding method was developed by controlling mixing ratios between the bonded couple. ${ }^{11}$ The second method was used in this research due to its ease of application and cost-effectiveness. By doing this, various mixing couples were prepared and brought together for bonding. The couples are prepared by a molding method. With a different mixing ratio, uncured PDMS was poured into a leveled 1 -in. ${ }^{2}$ plastic holder and cured at 65-deg C. When cured, two PDMS parts with different mixing ratios are put together under pressure to make full contact. The bonding was realized after heating the contacted couples at $80-\mathrm{deg} \mathrm{C}$ for $1.5 \mathrm{~h}$ under a weight of 125 g. $^{11}$

\subsection{Mixing and Curing Process}

PDMS specimens were prepared with seven different mixing ratios: $1: 2,1: 5,1: 7.5,1: 10,1: 12.5,1: 20$, and 1:50 (ratio of $B: A$ ). Parts $A$ and $B$ were mixed with these ratios and the mixture was cast into a $1-$ in. ${ }^{2}$ mold. The PDMS was cured under heat at 65-deg $\mathrm{C}$ after degassing. The heating process was performed on a leveled stage to make sure the cured PDMSs are uniform in thickness. Then cured PDMS was cut into samples with a size of $2 \times 10 \times 1 \mathrm{~mm}$ after peeling it off the mold. Using the same seven different mixing ratios, another set of samples were prepared for mechanical testing with a geometric effect. For each test, two samples with the same mixing ratio but different thicknesses were used. The thick sample is made by casting PDMS into a 1 -in. ${ }^{2}$ mold and peeling it off when it is fully cured. The thinner PDMS membrane sample was prepared using a spin-coating method. By manipulating the spin speed, the PDMS's thickness can be as thin as 20 to $30 \mu \mathrm{m}$. Cutting the two samples into the same size, $2 \times 10 \mathrm{~mm}$, is manually performed with care. Then, four couples formed with different mixing ratios (Table 2) were prepared for the bonding strength test.

\subsection{Test Frame and Test Procedure}

The mechanical properties were characterized using a MTS Tytron 250 microforce tester system, as shown in Fig. 1. The tester has a resolution in force of $10 \mu \mathrm{N}$ and an accuracy of $0.1 \mu \mathrm{m}$ in displacement measurement. The test specimen was mounted onto a double clapped gripper and held firmly as shown in Fig. 1. After the installation, a tensile force was applied to the specimen. The elongation and the corresponding force during the test were recorded 




Fig. 2 Test procedure. Mounted sample is pulled under the force from MTS system until it is broken. All the force and displacement data will be recorded by the computer and saved as an Excel file for future analysis.

automatically by MTS's BASIC TESTWARE. Figure 2 demonstrates the test procedure. All samples prepared as discussed above were mounted on the MTS system and tested.

\section{Results and Discussion}

\subsection{Mechanical Properties of PDMS with Different Mixing Ratios}

After recording the force and the elongation of each tested specimen, the stress and the corresponded strain can be derived. A stress-strain relationship of a PDMS specimen fabricated is presented in Fig. 3. At the beginning of the test, the resulting strain and stress have a linear relationship, which shows an elastic nature of the PDMS, a silicone rubber material. The Young's modulus was derived from the slope of the stress-strain curve. However, PDMSs with different weight mixing ratios behaved quite differently. Figure 4 and Table 3 show the change of Young's modulus versus the weight mixing ratios tested. It can be clearly

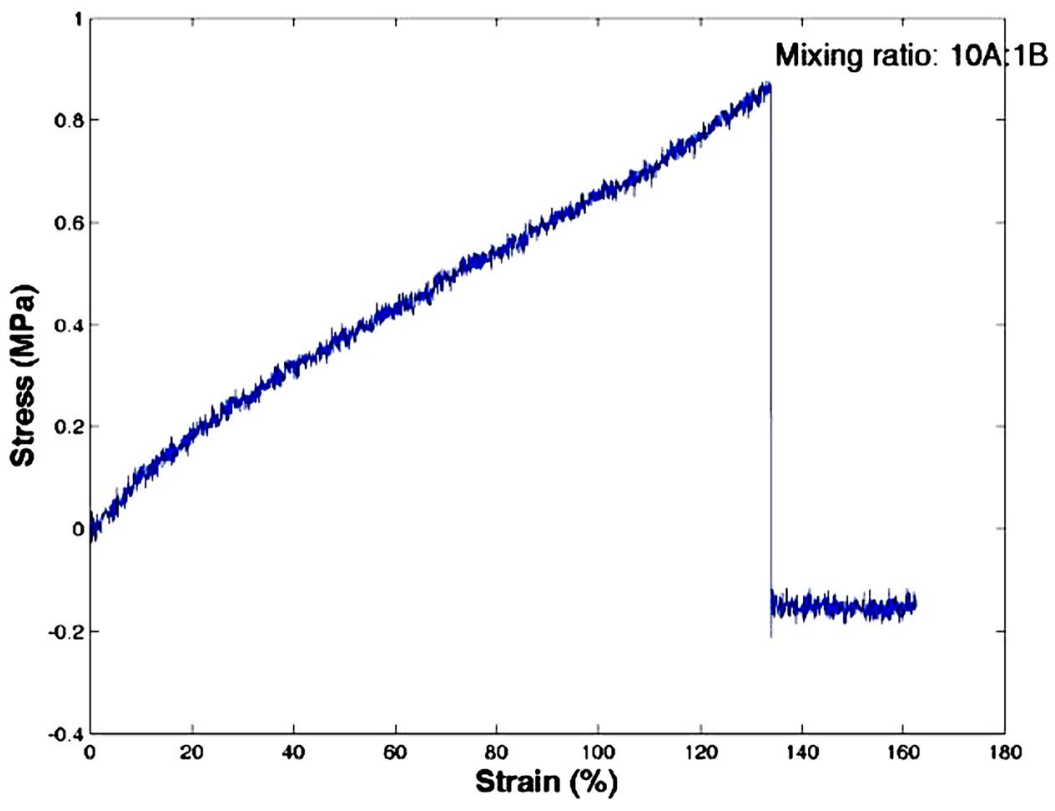

Fig. 3 Stress-strain relationship of PDMS with mixing ratio $10 A: 1 B$. 


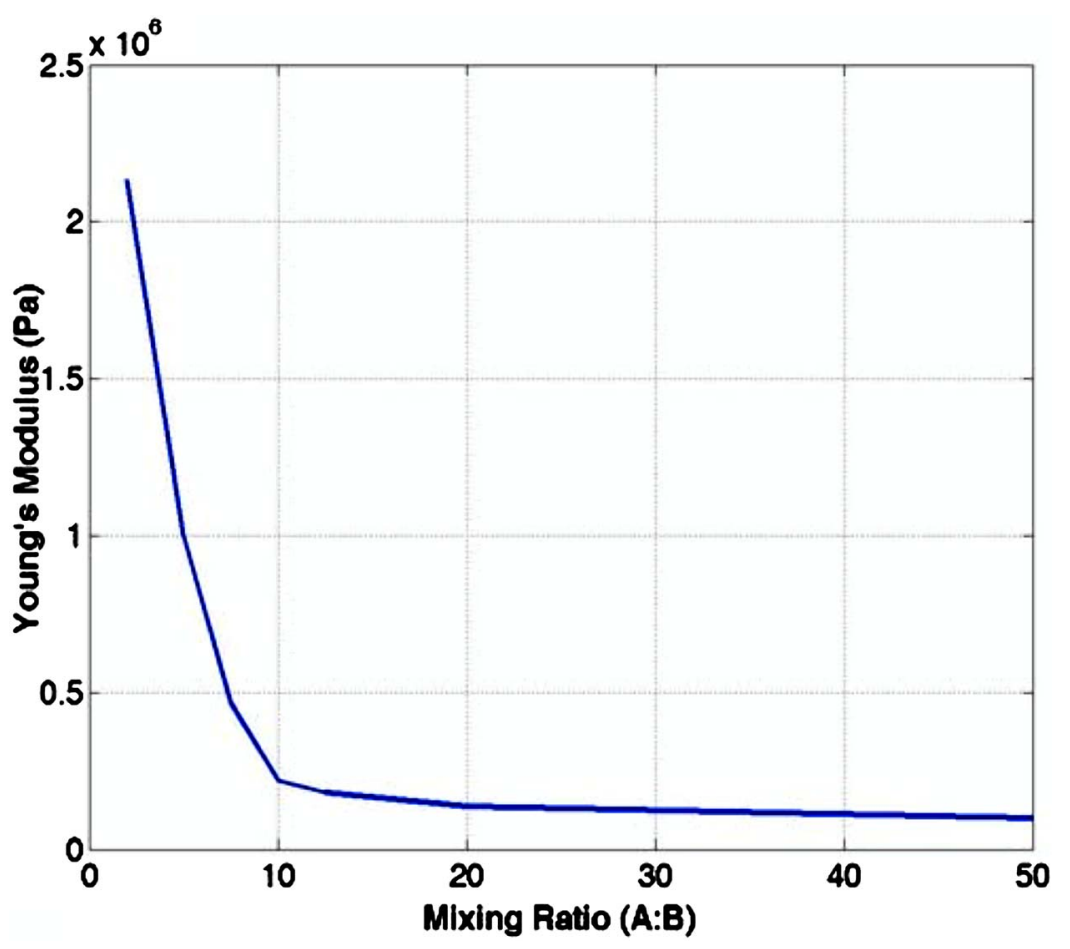

Fig. 4 Young's modulus versus weight mixing ratio.

seen from this curve that the Young's modulus decreases as the ratio of $A: B$ increases. This means the higher the portion of part $A$ in the PDMS, the lower the resulting Young's modulus (the softer the nature). This should be considered as the contribution of the polymerization of the materials: the larger the ratio, the more unreacted base polymer presented and the softer the final stage materials. Interestingly, the Young's modulus drops dramatically when the part $A$ ratio is relatively small $(A: B<10)$. For example, it decreases from 2.1 to $0.6 \mathrm{MPa}$ when the mixing ratio is increased from 2 to 10 . However, when the weight mixing ratio is greater than 10, the resulting Young's modulus of PDMS is almost independent of the mixing ratios (Fig. 4). When the mixing ratio increased from 10 to 50, the change in Young's modulus was approximately $0.5 \mathrm{MPa}$ only.

Table 3 Derived Young's modulus versus mixing ratios.

\begin{tabular}{lc}
\hline \hline Weight Mixing Ratio $(B: A)$ & Young's Modulus $(\mathrm{KPa})$ \\
\hline 2.00 & 2133 \\
5.00 & 1565 \\
7.50 & 1396 \\
12.50 & 405.4 \\
10.00 & 610.8 \\
20.00 & 238.2 \\
50.00 & 99.35 \\
\hline \hline
\end{tabular}

\subsection{Mechanical Properties with Different Thicknesses}

Scaling law happens on stiffer materials such as silicon in the MEMS community, as well as nickel and nickel alloys. A question may be asked if PDMS has a similar issue. To answer this question, a mechanical test was conducted to find the relationship between mechanical properties and the PDMS's thickness. The derived Young's modulus versus PDMS's $(12.5 A: 1 B)$ thickness is presented in Fig. 5. As shown in Fig. 5, the Young's modulus of PDMS with a thickness of $100 \mu \mathrm{m}$ is more than twofold greater than that of a PDMS with a thickness of $1.8 \mathrm{~mm}$.

\subsection{Bonding Strength of PDMS}

As shown in Table 2, four pairs of bonded PDMS were selected for the bonding test. All specimens were prepared under the same conditions (heated at 80-deg $\mathrm{C}$ for $1.5 \mathrm{~h}$ right after the curing process for individual part). After bonding, specimens were cut from these bonded PDMS couples in a way that the bonded interface was in the middle vertical to the loading direction, as shown in Fig. 6, and the specimens' sizes are both $2 \times 10 \times 3 \mathrm{~mm}$, which results in the bonding surface of the two coupled PDMS being right at the middle, which helps to eliminate the error caused by sloped mounting. Due to the difference of concentration at two sides of the bonding pieces, a diffusion layer is formed at the contact surface, as magnified in Fig. 6. It is indicated that the larger portion of part $A$, the thicker the bonding diffusion layer that is formed. The cut specimens were mounted to the MTS test system in a way that the bonded interface was perpendicular to the loading direction. A tensile force was then applied to the specimen continuously until the specimen was broken. The bonding 


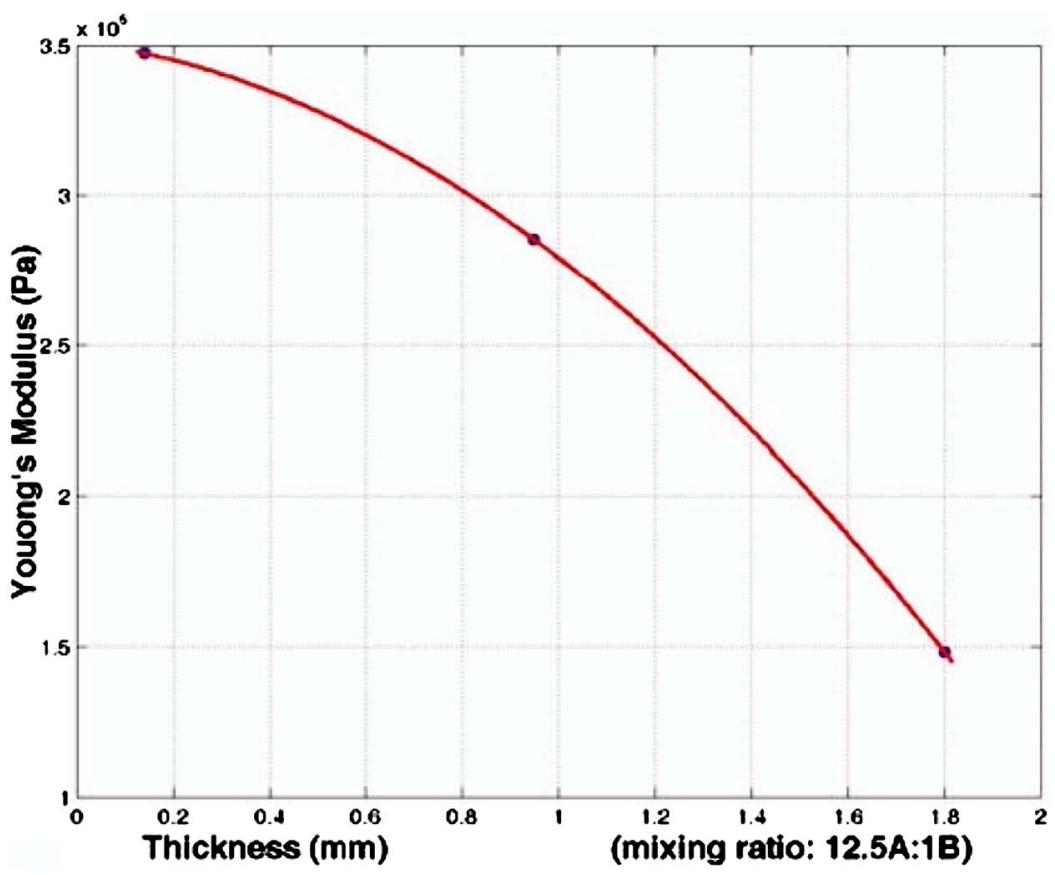

Fig. 5 Young's modulus versus PDMS thickness.

strength versus mixing ratios is shown in Fig. 7. It is indicated that the bonding strength strongly depends on the mixing ratio. The bonding strength increases first as the mixing ratio is increased. However, a maximum bonding strength of $130 \mathrm{kPa}$ occurred on the bonded couple between mixing ratios $30 A: 1 B$ and $3 A: 1 B$. After this transition point, the resulting bonding strength decreases as the ratio of part $A$ relative to part $B$ increases (Fig. 7). After examining the broken specimens, it was found that the fracture surface did not occur at the bonded interface. Instead, it occurred at the side with the larger portion of part $A$ and close to the bondedinterface. This can be explained by the fact that the diffusion layer formed at the bonded interface. From Fig. 5 we know that the larger portion of part $A$ will result in a soft natured PDMS. The diffusion layer has higher concentration of agent part $B$, and thus a relatively smaller portion of part $A$, in comparison to the broken part. Therefore, the resulting PDMS of this thin layer is rela- tively stiffer. The diffusion layer has a lower concentration of agent part $B$ in comparison to the unbroken part. Therefore, the bonded interface has an intermediate mechanical stiffness due to the diffusion.

\section{Conclusion}

We have demonstrated that by controlling the mixing ratio of PDMS's two components (part $A$ and part $B$ ), different mechanical properties of PDMS can be achieved. Young's modulus decreases as the mixing ratios increase. There is a transitional mixing ratio (part $A$ :part $B=10$ ) after which the Young's modulus is almost independent of the mixing ratio tested. PDMS's thickness plays an important role in determining mechanical properties. The thinner the PDMS, the stiffer it behaves along its normal direction. The bonding strength between PDMS couples with different mixing ratios shows that it depends on the mixing ratio. A maximum
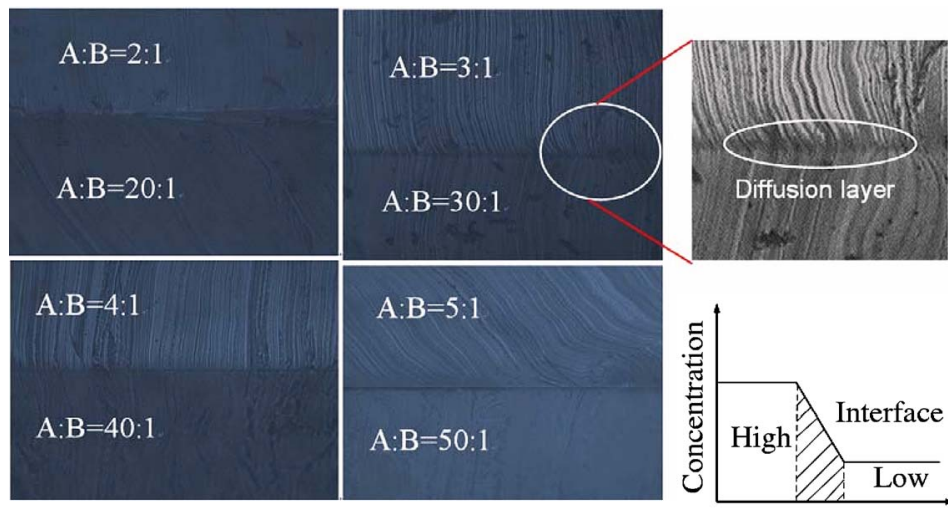

Location

Fig. 6 Cross-sectional view of bonded PDMS couples with different mixing ratios and diffusion layer between bonding parts. 


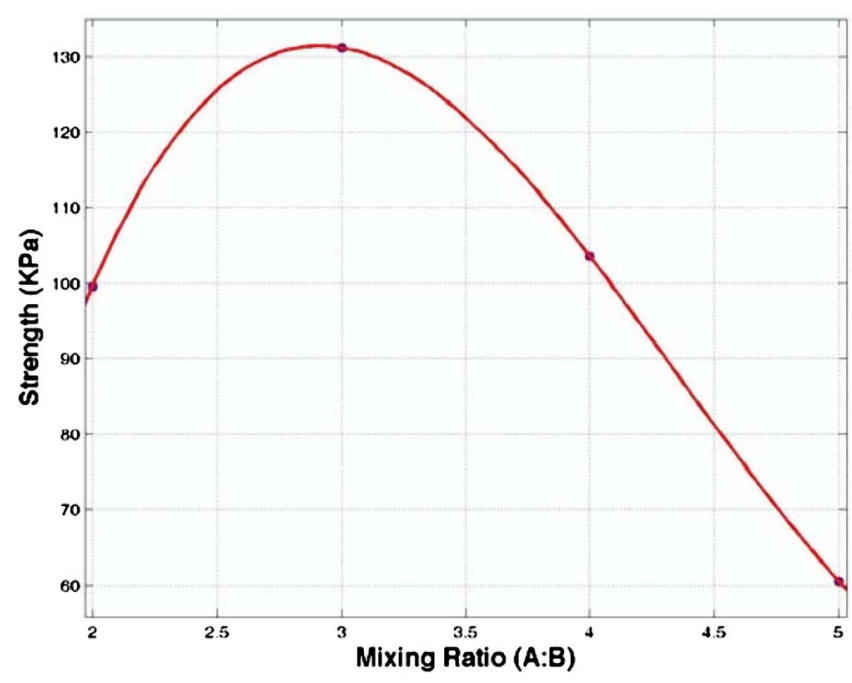

Fig. 7 Bonding strength versus mixing ratios of bonded couples.

bonding strength of $130 \mathrm{kPa}$ occurred on a bonded couple between mixing ratios of $30 A: 1 B$ and $3 A: 1 B$. The fracture of bonded PDMS does not occur at the bonding interface; it occurs at the side with large portion of part $A$. This implies that the interface is not the weakest location, due to the fact that intermediate material property is formed by the diffusion between the bonded couple. This featured property is good and important for device design, because it provides the opportunity to tailor it in designing devices by controlling the concentration of PDMS, and the bonded interface is free of concern in terms of reliability or deformation. On the other hand, for actuation purposes, one has to pay attention to the properties at the interface. The good news is that the bonded interface forms a graded material that links two parts together continuously with intermediate mechanical properties. For multilayer structures, this feature is also important because it is possible to reinforce the structure's property by alternating the arrangement of bonded layers. These results will be very helpful to assist the design and fabrication of various MEMS devices using PDMS for biological applications.

\section{Acknowledgments}

The work was supported by the Florida Space Research Initiative program.

\section{References}

1. B. Michel, A. Bernard, A. Bietsch, E. Delamarche, M. Geissler, D. Juncker, H. Kind, J.-P. Renault, H. Rothuizen, H. Schmid, P. Schmidt-Winkel, R. Stutz, and H. Wolf, "Printing meets lithography: Soft approaches to high-resolution patterning," IBM J. Res. Dev. 45(5), 697-719 (2001).

2. G. M. Whitesides, E. Ostuni, S. Takayama, X. Jiang, and D. E. Ingber, "Soft lithography in biology and biochemistry," Аnпи. Rev. Biomed. Eng. 3, 335-373 (2001).
3. D. S.-W. Park, K. Kim, B. Pillans, and J.-B. Lee, "Polydimethylsiloxane-based pattern transfer process for the post-IC integration of MEMS onto CMOS chips," J. Micromech. Microeng. 14, 335-340 (2004).

4. J.-H. Kim, K.-H. Na, C. J. Kang, and Y.-S. Kim, "A disposable thermopneumatic-actuated micropump stacked with PDMS layers and ITO-coated glass," Sens. Actuators, A 120(2), 365-369 (2005).

5. A. Yamaguchi, P. Jin, H. Tsuchiyama, T. Masuda, K. Sun, S. Matsuo, and H. Misawa, "Rapid fabrication of electrochemical enzyme sensor chip using polydimethylsiloxane microfluidic channel," Anal. Chim. Acta 468(1), 143-152 (2002).

6. T. Fujii, "PDMS-based microfluidic devices for biomedical applications," Microelectron. Eng. 61-62, 907-914 (2002).

7. Y. S. Shin, K. Cho, S. H. Lim, S. Chung, S.-J. Park, C. Chung, D.-C. Han, and J. K. Chang, "PDMS-based micro PCR chip with Parylene coating," J. Micromech. Microeng. 13, 768-774 (2003).

8. L. Ceriotti, N. F. de Rooij, and E. Verpoorte, "An integrated fritless column for on-chip capillary electrochromatography with conventional stationary phases," Anal. Chem. 74(3), 639-647 (2002).

9. X. Yu, D. Zhang, T. Li, L. Hao, and X. Li, "3-D microarrays biochip for DNA amplification in polydimethylsiloxane (PDMS) elastomer," Sens. Actuators, A 108, 103-107 (2003).

10. GE silicones, RTV 615 datasheet.

11. M. A. Unger, H.-P. Chou, T. Thorsen, A. Scherer, and S. R. Quake, "Monolithic microfabricated valves and pumps by multilayer soft lithography," Science 288, 113-116 (2000).

12. X. Yang, C. Grosjean, Y.-C. Tai, and C.-M. Ho, "A MEMS thermopneumatic silicone rubber membrane valve," Sens. Actuators, A 64(1), 101-108 (1998).

13. B. Yang, J. W. Levis, and Q. Lin, "A PDMS-based constant-flowrate microfluidic control device," 17th IEEE Int. Conf. Micro Electro Me chanical Systems (MEMS), pp. 379-382 (2004).

14. B. T. Ginn and O. Steinbock, "Polymer surface modification using microwave-oven-generated plasma," Langmuir 19, 8117-8118 (2003).

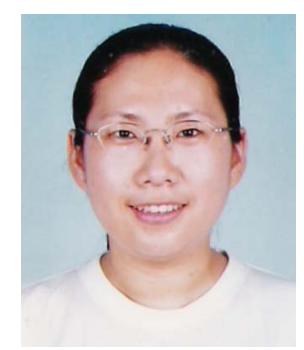

Miao Liu is currently a PhD candidate in the Mechanical, Materials and Aerospace Engineering Department of University of Central Florida. Ms. Liu received both her Masters and Bachelor degree from Tsinghua University in 2002 and 2000, respectively. Her current research toward her $\mathrm{PhD}$ degree is mainly about microfluidic systems for BioMEMS. Her research interests include MEMS design, microfabrication, system integrations and characterization, actuators, microfluidic systems, mechanical sensors, Bio-MEMS, finite element analysis (FEM) and CAD/CAM.

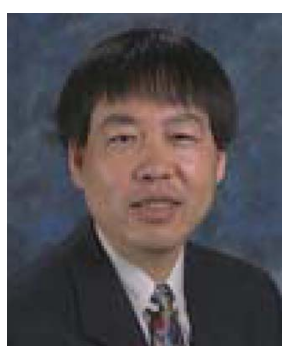

Quanfang Chen is currently an associate professor and the Program Coordinator of Miniature Engineering Systems at the Mechanical, Materials and Aerospace Engineering Department of University of Central Florida (UCF). He is also an affiliated faculty of Advanced Materials Processing and Analysis Center (AMPAC), and the Director of MEMS and Nanomaterials Laboratory at UCF. Dr. Chen received his PhD from Tsinghua University in 1989. Before joining UCF, he was an assistant research professor (equivalent) at University of California at Los Angeles (UCLA). His research interests include microscale and nanoscale fabrications, multifunctional nanocomposite for MEMS/NEMS, sensors using nanostructural materials, actuators including hybrid compact actuators, microfluidic systems (valves and pumps) for Bio-MEMS, micro/nano mechanics including friction and control and molecular dynamic simulations. 